\title{
Present Bias and Collective Dynamic Choice in the Lab
}

\author{
By MatThew O. JaCKSON ANd LeEAT Yariv*
}

\begin{abstract}
We study collective decisions by time-discounting individuals choosing a common consumption stream. We show that with any heterogeneity in time preferences, utilitarian aggregation necessitates a present bias. In lab experiments three quarters of "social planners" exhibited present biases, and less than two percent were time consistent. Roughly a third of subjects acted as if they were pure utilitarians, and the rest chose as if they also had varying degrees of distributional concerns. (JEL C91, D12, D71, D72)
\end{abstract}

Many important decisions over sequences of consumption or budget allocations are made by groups of decision makers: households, political committees, boards of firms, or even different motives within one individual. If members of a group differ in their time preferences, a tension arises when making collective intertemporal choices. We explore how this tension distorts collective intertemporal decisions.

Indeed, individuals vary in their time preferences. For example, in most parts of the world, women have significantly higher life expectancies than men: in the United States and the United Kingdom, current estimated life expectancies are 82 years for women and 78 for men, with similar numbers of 85 and 79 in France and Spain; 74 and 62 in Russia; 76 and 72 in China; 87 and 80 in Japan; 77 and 70 in Brazil; and 54 and 52 in South Africa. ${ }^{1}$ This is amplified by age differences between men and women at marriage. In the United States, men are typically several years older than their wives. ${ }^{2}$ When translated into discount factors, this suggests that husbands and wives might discount the value of savings at substantially different rates.

Despite this heterogeneity, the foundations of classical economics assume that firms and other organizations act as "representative agents," and that their behavior

\footnotetext{
* Jackson: Department of Economics, 579 Serra Mall Stanford University, Stanford, CA 94305 the Santa Fe Institute, and CIFAR; (e-mail: jacksonm@stanford.edu); Yariv: Division of the Humanities and Social Sciences, Caltech, MC 228-77 Pasadena, CA 91125 (e-mail: lyariv@hss.caltech.edu). We thank Nageeb Ali, Sandro Ambuehl, James Andreoni, Kenneth Arrow, Mariagiovanna Baccara, Miguel Angel Ballester, Douglas Bernheim, Martin Browning, Christopher Chambers, Jeff Ely, Keith Ericson, Drew Fudenberg, Jerry Green, Olivier l'Haridon, Andrew Hertzberg, Julian Jamison, Lauren Merrill, Jochen Mierau, Massimo Morelli, Efe Ok, Andrew Postlewaite, Antonio Rangel, Ariel Rubinstein, Erik Snowberg, and Tomasz Strzalecki for useful discussions and comments. We also thank the anonymous referees for helpful suggestions. Jan Zapal provided us with superb research assistance. We gratefully acknowledge financial support from the National Science Foundation (SES 0551014 and SES 0961481) and the Gordon and Betty Moore Foundation. The authors declare that they have no relevant or material financial interests that relate to the research described in this paper.

Go to http://dx.doi.org/10.1257/aer.104.12.4184 to visit the article page for additional materials and author disclosure statement(s).

${ }^{1}$ Expectancies are from birth for children born from 2010 to 2015, see the United Nations Statistics Division Social Indicators, updated in December 2010, based on data from the World Population Prospects: The 2008 Revision (CD-ROM Edition), supplemented by official national statistics published in the United Nations Demographic Yearbook 2008.

${ }^{2}$ Between 1947 and 2010, a groom was, on average, 2.3 years older than his bride (see Drefahl 2010). Browning
} 
is time consistent. Operationally, individuals, alone and in groups, evaluate streams of consumption using an instantaneous utility function and discount each period's instantaneous utility in an exponentially decaying manner. This assumption persists even though it was recognized long ago, by Marglin (1963) and Feldstein (1964), that it is difficult to derive an aggregate time independent discount rate for a planner facing a society of heterogeneous agents. More recently, Gollier and Zeckhauser (2005) showed that a representative agent will have a time-varying discount factor if there is sufficient uncertainty and heterogeneity in the environment. ${ }^{3}$

Our Proposition 1 formalizes an intuition seen in the Gollier-Weitzman (2010) puzzle. Gollier and Weitzman argue that an appropriate collective discount factor should exhibit more patience for decisions that are further in the future. Our Proposition 1 shows that there is necessarily a present bias in utilitarian decision making. Specifically, if members of a group have heterogeneous discount factors, then maximizing a weighted sum of their utility functions leads to a present-bias: the group will not only prefer early to later consumption, but will also appear increasingly patient as decisions are pushed into the future. In fact, for a uniform distribution of discount factors in the population, maximizing the sum of utilities translates into hyperbolic discounting.

The difficulties of time consistency are not unique to utilitarian weighting. As shown by Zuber (2011), having stationary collective preferences over (private) consumption streams requires that all agents have the same discount factor. That result does not imply that the same would be true when aggregating preferences over common consumption streams, as there is less conflict between agents given that all prefer more sooner to less later and are consuming the same stream. Nonetheless, as we show in a companion paper (Jackson and Yariv 2014), every non-dictatorial aggregation method is time inconsistent if there is any discounting heterogeneity within the population.

The following simple example illustrates why present-bias is inevitable. Two time-consistent individuals, Constantine and Patience, choose their household consumption by maximizing their joint welfare. Constantine has a discount factor of 0.5 , while Patience has a discount factor of 0.8 . Both experience identical instantaneous utility. When Constantine and Patience are considering 10 utiles today relative to 15 utiles tomorrow, they are comparing a total collective utility of $10+10=20$ with $15(0.8+0.5)=19.5$. They therefore jointly choose the immediate 10 utiles. Suppose now that Constantine and Patience compare 10 utiles at time $t \geq 1$ and 15 utiles at time $t+1$. Now, the comparison is between a total collective utility of $10\left(0.8^{t}+0.5^{t}\right)$ and $15\left(0.8^{t+1}+0.5^{t+1}\right)$. For instance, if $t=1$, the comparison is

\footnotetext{
(2000), using Canadian data finds that the wife of a 65-year-old man would have, on average, an approximately 50 percent longer expected survival horizon than her husband. See also Schaner (2013) for evidence on differences in time preferences inside households in Western Kenya. Kuhn (2013) examines household consumption and expenditure data to test the theory we provide here, finding that more diverse households suffer more severe time-inconsistency. We discuss further related literature below.

${ }^{3}$ Recent work in the context of household decisions, such as Bernheim (1999); Browning (2000); Mazzocco (2007); Xue (2008); Abdellaoui, l'Haridon, and Paraschiv (2010); and Hertzberg (2012) considers the implications of preference heterogeneity on intertemporal consumption decisions under particular aggregation protocols. That literature suggests, for instance, that households may have hyperbolic preferences (Hertzberg 2012) and that commitment devices may play a role in determining consumption patterns (in Mazzocco 2007; and Schaner 2012); see also Weitzman (2001); Caplin and Leahy (2004); Blackorby, Bossert, and Donaldson (2005); Jamison and Jamison (2011); Farmer and Geanakoplos (2009); and Rode (2011).
} 
between 13 and 13.35, and the delayed consumption leads to greater total utility. In fact, for any $t \geq 1$, the total utility would be larger from the delayed consumption of 15 utiles, but the immediate decision exhibits the reverse. The household's behavior is time-inconsistent and present biased: the household prefers an immediate reward of 10 to a delayed reward of 15 , but reverses its preference whenever rewards are both pushed into the future.

After showing that a present-bias is inevitable when maximizing some weighted sum of utilities, we turn to our main focus: a series of laboratory experiments in which one subject chooses a consumption stream for other subjects, who differ in their discount factors. In other words, subjects make choices as if they are social planners aggregating the preferences of several other individuals. Three main insights emerge.

- Fifty-nine out of 60 subjects are time inconsistent. Three quarters of the subjects are present-biased, and the remaining time-inconsistent subjects exhibit either future bias or situation-based inconsistencies. ${ }^{4}$

- Fitting collective utility functions for the planners that combine utilitarian with egalitarian motives enables us to match more than 80 percent of the 38 choices they each made.

- Subjects differ and can be classified according to how they weight utilitarian and egalitarian motives. Just under one-third of subjects act as if they are pure utilitarians, while the remaining two-thirds act as if they also weight inequality (negatively). Subjects who place the greatest weight on equality exhibit mixed time inconsistencies, and those weighting equality less exhibit present bias, with most (about two-thirds of) subjects acting as if they weight equality slightly but noticeably. ${ }^{5}$

Our experiments are based on a (new) method of inducing discounting without relying on timing. Instead of delaying payments, we make all payments at the same time, but induce discounting via tokens of different types (mimicking dates) and then discount the tokens by different amounts. For example, suppose the induced discount factor is 0.9 . When choosing a sequence of consumptions of $(200,50,100)$ of three respective types of tokens, the subject receives a payment of $200+45+81$ experimental cents. This method allows us to directly control the discount factor of the subjects and avoids confounding factors (e.g., uncertainty) that enter subjects' evaluations of actual delayed payment and are a challenging confound in traditional experiments. The trade-off is that although our technique allows us to control and impose discount factors, subjects are not facing actual time discounted consumption utilities - so our experiments are a test of how people aggregate sequences of payments that involve discounting, but not a direct test of how people behave when faced with time-based choices. This also allows us to isolate the aggregation issue

\footnotetext{
${ }^{4}$ As we show in Jackson and Yariv (2014), other sorts of time inconsistencies are induced by non-utilitarian collective decision making. For example, in the decisions studied in the experiments, purely egalitarian motives can generate a future bias. Mixed utilitarian and egalitarian motives can generate a combination of present and future biased decisions.

${ }^{5}$ Those two thirds of the subjects' choices diverge from a pure utilitarian decision only when the standard deviation in payoffs is more than three times the difference in average payoffs across decisions.
} 
by imposing actual exponential discounting on the base preferences and then examine biases that result in the aggregation.

Before turning to the main body of the paper, we make three further remarks. First, our results provide a potential explanation for why individual decision makers may exhibit a present bias: an individual aggregating a group of internal but diverse preferences could be time inconsistent. Indeed, time inconsistent models of decision-making appear to explain well a variety of real-world phenomena, ranging from saving behavior (Laibson 1997 and Beshears et al. 2008) to physical exercise (DellaVigna and Malmendier 2006). 6 The idea that individuals might be thought of as having some internally conflicting preferences appears in a variety of places, possibly the most related of which is the recent paper by Green and Hojman (2009) that provides a welfare analysis using revealed-preferences in such settings. ${ }^{7}$ A contribution of the current paper is the insight that viewing individuals as non-degenerate utilitarian collectives leads necessarily to behaviors exhibiting time inconsistency and a present bias.

Second, our experimental analysis is related to that of Engelmann and Strobel (2004), who considered allocation decisions in which agents made decisions "behind the veil of ignorance." Their subjects made decisions affecting outcomes for a set of possible future roles and were paid according to a randomly selected role. Two main differences between our experiments and theirs are as follows. First, our allocations are designed to detect inconsistencies in time preferences and to distinguish between their variations (present-bias, future-bias, etc.). Second, we identify preferences of social planners, rather than general attitudes toward bargaining outcomes, and in our setting experimental planners make choices that only affect others. 8

Finally, our analysis allows us to estimate the social preferences exhibited by different subjects and we see substantial and statistically significant heterogeneity in those preferences, including some utilitarian concerns and some equity concerns. These illuminate a debate regarding subjects' utilitarian and egalitarian motives (e.g., see Charness and Rabin 2002; Andreoni and Miller 2002; and Fisman, Kariv, and Markovits 2007).

\footnotetext{
${ }^{6}$ The full literature documenting time inconsistencies is too vast to cover here. Some seminal references include Herrnstein (1961) and Thaler (1981). These phenomena seem fundamental in that they are observed in species other than humans as well (e.g., rats and pigeons as in Ainslie 1975 and Rachlin 2000). For some background, see, e.g., Frederick, Loewenstein, and O'Donoghue (2002). Behavior nonetheless depends on context: when faced with very simple monetary decisions in the lab, Andreoni and Sprenger (2012a, b) find that many individuals appear to be time consistent.

${ }^{7}$ Emerging neuro-scientific evidence suggests that some aggregation of motives that respond differently to timed rewards, goes on in an individual's brain (e.g., see Hare, Camerer, and Rangel 2009; McClure et al. 2004, 2007; and Glimcher and Rustichini 2004). Notable models with multiple motives of agents include, among others: Thaler and Shefrin (1981); O'Donoghue and Rabin (1999); Bernheim and Rangel (2004); Bénabou and Tirole (2005); Amador, Werning, and Angeletos (2006); Brocas and Carrillo (2008); Fudenberg and Levine (2006); Ambrus and Rozen (2014); and Cherepanov, Feddersen, and Sandroni (2013). In many of those settings, differences in preferences lead to a conflict between, e.g., current and future selves. In contrast, we focus on how multiple selves collectively make choices.

${ }^{8}$ When agents are randomly paid according to a role as in Engelmann and Strobel (2004), an expected utility maximizer would choose alternatives maximizing the sum of expected utilities. Indeed, utilitarian motives appear quite strongly in the Engelmann and Strobel experiments. Our design does not necessarily induce utilitarian motives as the decision maker is making decisions for others.
} 


\section{The Setting}

A set $N=\{1, \ldots, n\}$ of agents make a collective decision over streams of consumption. Agents all consume the same stream, denoted by $C=\left(c_{1}, c_{2}, \cdots\right)$, where each $c_{t} \in[0,1] .9$

Agents maximize a time additive discounted utility function. Agent $i$ has a discount factor $\delta_{i} \in(0,1)$ and an increasing and twice continuously differentiable instantaneous utility function $u_{i}:[0,1] \rightarrow \Re$ such that a stream $C=\left(c_{1}, c_{2}, \ldots\right)$ is evaluated as ${ }^{10}$

$$
U_{i}(C)=\sum_{t} \delta_{i}^{t} u_{i}\left(c_{t}\right)
$$

Let $\mathcal{U}$ denote the set of possible preferences $\left(\delta_{i}, u_{i}\right)$ satisfying the conditions above. ${ }^{11}$

A society of $n$ individuals is denoted by $\left(\delta_{1}, u_{1} ; \ldots \delta_{n}, u_{n}\right)$. We sometimes abuse notation and let $U_{i}$ denote the corresponding $\left(\delta_{i}, u_{i}\right)$, so that a society can be denoted by $U=\left(U_{1}, \ldots, U_{n}\right) \in \mathcal{U}^{n}$.

We emphasize that requiring individuals to consume the same stream (such as a household deciding on how to budget consumption across time) leads to stronger results, as further allowing individuals to decide on how to divide up consumption among themselves exacerbates conflicts in preferences making inconsistencies easier to derive. ${ }^{12}$

\section{Utilitarian Aggregation of Preferences and Present Bias}

We begin by showing that the most prominent class of collective utility functions, weighted utilitarian functions, necessarily exhibits present bias; a sort of time inconsistency that matches some evidence on behavior.

A collective utility function is a weighted utilitarian function if there exist weights $w_{i} \in[0,1]$ such that $\sum_{i} w_{i}=1$ and

$$
V(C)=\sum_{i} w_{i} U_{i}(C) .
$$

The weights $w_{i}$ can correspond to the fraction of the population with each particular utility function, or to factors used to trace out the Pareto frontier. For example, if $w_{i}$ is proportional to $\frac{1-\delta_{i}}{n}$, then $V(C)$ corresponds to the average (normalized) discounted utilities across the population. Thus, $V(C)$ is often an object of interest in applied work.

\footnotetext{
${ }^{9}$ The assumption that timed consumption is uniformly bounded will assure that net present values are always well-defined (for well-behaved instantaneous utility functions). The assumption that it is bounded by 0 and 1 is without loss of generality. Finite-horizon problems can be considered via strings that have only finitely many positive entries.

${ }^{10}$ This specification of time discounted additively separable utility clearly precludes certain sorts of complementarities in consumption across periods. Nonetheless, it is still important to understand its properties as it is so standard in the literature, partly because of its time consistency properties and partly because the complementarities are often secondary.

${ }^{11}$ For notational convenience, we ignore the normalization factors such as dividing by $\left(\left\{1-\delta_{i}\right\}_{i}\right)$.

${ }^{12}$ See Zuber (2011) for an examination of settings in which all individuals consume their own streams.
} 
Utilitarian functions can lead to very familiar "non-standard" preferences, as the following two examples illustrate.

Example 1 (Hyperbolic Discounting): Consider a society with a continuum of agents ${ }^{13}$ with $\delta_{i}=i$, where $i$ is uniformly distributed on $(0,1)$. All agents share an identical instantaneous utility function, $u_{i}=u$.

In this simple case, the resulting utilitarian collective utility function is

$$
V(C)=\sum_{t} \int \delta_{i}^{t} u\left(c_{t}\right) d i=\sum_{t} \int i^{t} u\left(c_{t}\right) d i
$$

or

$$
V(C)=\sum_{t} \frac{u\left(c_{t}\right)}{1+t}
$$

Thus, a society with uniformly distributed discount factors generates a utilitarian collective utility function that is exactly hyperbolic. ${ }^{14,15}$

Example 2 (Fixed Costs of Delay): Consider a society of two types of agents. One type of agent is completely impetuous: $\delta_{1}=0$ and $u_{1}\left(c_{t}\right)=a$ for any $c_{t}>0.16$ This type of agent simply wants immediate gratification, and is insensitive to the amount of immediate consumption. The other type of agent is standard, with a discount factor $\delta_{2}>0$ and instantaneous utility $u_{2}$, an increasing function. Let $\lambda$ be the proportion of impetuous agents. The resulting utilitarian collective function is

$$
V(C)=\sum_{t} \lambda \delta_{1}^{t} u_{1}\left(c_{t}\right)+(1-\lambda) \delta_{2}^{t} u_{2}\left(c_{t}\right)
$$

or

$$
V(C)= \begin{cases}\lambda a+(1-\lambda) \sum_{t} \delta_{2}^{t} u_{2}\left(c_{t}\right) & \text { if } c_{1}>0 \\ (1-\lambda) \sum_{t} \delta_{2}^{t} u_{2}\left(c_{t}\right) & \text { otherwise }\end{cases}
$$

This society exhibits a collective utility function that has a fixed cost of delaying any immediate consumption, but exhibits exponential discounting thereafter, matching the model and array of experiments presented by Benhabib, Bisin, and Schotter (2010).

\footnotetext{
${ }^{13}$ This moves outside of our finite model, but can be easily approximated with finite settings.

${ }^{14}$ We note that we did not normalize each agent's utility (with the factors $\left\{1-\delta_{i}\right\}$ ). In terms of normalized discounted utility, this formulation effectively puts greater weight on agents that are more patient. Normalizing provides a variation on the functional form, but with similar induced behavior.

${ }^{15}$ This example is reminiscent of a model analyzed by Souzou (1998), who illustrated how uncertainty over exponential hazard rates can generate hyperbolic discounting. Relatedly, Dasgupta and Maskin (2005) noted that uncertainty about future rewards may translate into an apparent present-bias. Xue (2008) shows that aggregation in a two person model can exhibit some quasi-hyperbolic features.

${ }^{16}$ Again, this sits at the extreme of our model as we assume that $\delta_{i}>0$ and $u_{i}$ is increasing, but is easily approximated within the model.
} 
As we now show, a present bias is inherent to any utilitarian aggregation of heterogeneous time preferences.

Let $C_{x}^{t}$ denote a consumption stream with $c_{t}=x$ and $c_{t^{\prime}}=0$ for $t^{\prime} \neq t$.

Present-biased Collective Utility Functions.-A collective utility function is present biased if:

- $V\left(C_{x}^{t}\right) \leq V\left(C_{y}^{t^{\prime}}\right)$ implies $V\left(C_{x}^{t+1}\right) \leq V\left(C_{y}^{t^{\prime}+1}\right)$ for any $x, y$, and $t^{\prime}>t \geq 1$, but the reverse implication sometimes fails:

- For any $t^{\prime}>t \geq 1$ there exist $x$ and $y$ such that $V\left(C_{x}^{t+1}\right)<V\left(C_{y}^{t^{\prime}+1}\right)$ while $V\left(C_{x}^{t}\right)>\bar{V}\left(C_{y}^{t^{\prime}}\right)$.

Present bias indicates more impatience as the relevant consumption becomes more immediate. The first part of the definition states that if one level of consumption at some time $t^{\prime}$ is preferred to another at an earlier time $t$, then the same preference ordering holds when both consumptions are postponed, so that future preferences are at least as patient. The second part of the definition indicates that there exist some choices that reverse themselves over time: if offered today the immediate consumption is preferred, while if deferred to some point in the future the choice corresponds to more patience (a description going back to Strotz 1955 and corresponding to the impulsiveness suggested by Ainslie 1975).

PROPOSITION 1: If $u_{i}=u$ for all $i$ and $V$ is a weighted utilitarian function with weights $w_{i}>0$ and $w_{j}>0$ for at least two agents $i$ and $j$ such that $\delta_{i} \neq \delta_{j}$, then $V$ is present biased.

The intuition behind this result is straightforward. Suppose the group of $n$ agents is characterized by the sequence of discount factors $\delta_{1}<\delta_{2}<\cdots<\delta_{n}$. The effective discount factor of period $t$ consumption in the collective utility is given by $w_{1} \delta_{1}^{t-1}+w_{2} \delta_{2}^{t-1}+\cdots+w_{n} \delta_{n}^{t-1}$. For simplicity, assume that all the weights are positive, $w_{i}>0$ for all $i$. As $t$ increases,

$$
\frac{w_{1} \delta_{1}^{t}+w_{2} \delta_{2}^{t}+\cdots+w_{n} \delta_{n}^{t}}{w_{1} \delta_{1}^{t-1}+w_{2} \delta_{2}^{t-1}+\cdots+w_{n} \delta_{n}^{t-1}} \rightarrow \delta_{n}
$$

Thus, as $t$ grows agents with greater patience gain implicit importance in determining the rates of substitution across time, and the collective utility exhibits more patience, and hence a present bias. ${ }^{17}$

\footnotetext{
${ }^{17}$ The analysis carries over to an even more general class of collective utility functions that take as inputs the net present values assessed by the group of individuals, including collective utilities that result from Nash bargaining, which weight individual utilities multiplicatively. Indeed, for any (smooth) functional $F\left[U_{1}, \ldots, U_{n}\right]$, the corresponding marginal rate of substitution between periods $t$ and $t+1$ is $\frac{\sum_{i} \delta_{i}^{t} u^{\prime}\left(c_{t+1}\right) \frac{\partial F}{\partial U_{i}}}{\sum_{i} \delta_{i}^{t-1} u^{\prime}\left(c_{t}\right) \frac{\partial F}{\partial U_{i}}}$. As long as the sensitivity of $F$ to different agents is not too extreme, this will also converge to weighting only the most patient agents as $t$ grows. In Jackson and Yariv (2014) we show that time inconsistency is endemic to all non-dictatorial rules that respect unanimity.
} 
The proposition implies that whenever a heterogeneous group of individuals (or a collection of temporal motives within one individual) determines choices by selecting a Pareto efficient alternative (maximizing a weighted sum of individual utility functions), a present bias ensues.

Averaging discount factors has further implications relevant for modeling representative agents as well as for econometric estimations of preferences. As noted by a number of authors following Marglin (1963) and Feldstein (1964), a weighted utilitarian function would appear to have a time-dependent exponential discount factor, where the value of the implicit discount factor at time $t$ is given by ${ }^{18}$

$$
\hat{\delta}_{t} \equiv\left[\sum_{i} w_{i} \delta_{i}^{t}\right]^{1 / t}
$$

We then see that this implicit discount factor increases with time: For $t_{1}>t_{2}$, given that $x^{t_{2} / t_{1}}$ is concave, it follows from Jensen's inequality that

$$
\hat{\delta}_{t_{2}}=\left[\sum_{i} w_{i} \delta_{i}^{t_{2}}\right]^{1 / t_{2}}=\left[\sum_{i} w_{i}\left(\delta_{i}^{t_{1}}\right)^{t_{2} / t_{1}}\right]^{1 / t_{2}} \leq\left[\sum_{i} w_{i} \delta_{i}^{t_{1}}\right]^{1 / t_{1}}=\hat{\delta}_{t_{1}} .
$$

Therefore, in concordance with the present bias that is innate to averaging of preferences, effective exponential discount factors increase with time. 19

\section{Collective Time Preferences in the Laboratory}

We conducted a set of experiments designed to elicit social preferences over joint consumption streams and to inspect whether social planners in the lab exhibit time inconsistency.

\section{A. A Methodological Point on Experiments with Time Preference}

There are several challenges in eliciting time preferences in the lab. First, explicitly delaying payments to subjects does not necessarily map into delayed changes in their consumption levels. Instead, the delays may simply affect their cash flow. Delayed payments can be substituted for by shifting existing wealth across time at the subject's interest rate on savings. Therefore, measured discount factors may simply reflect the interest rates and/or borrowing constraints that subjects face, which may be nonstationary. This concern is particularly strong when rewards are monetary.

Second, agents may be uncertain about their preferences for cash in the future, introducing risk and uncertainty as confounds, especially for college students who frequently face cash constraints and uncertainty about their expenses. ${ }^{20}$

\footnotetext{
${ }^{18}$ Estimating an average discount rate from a distribution $G$ on $[0,1]$ while not accounting for heterogeneity may lead an econometrician to assess the $t^{\prime}$ th moment of the distribution, $E_{G}\left(\delta^{t}\right)$, rather than the expected discount to the $t$ th power, $\left[E_{G}(\delta)\right]^{t}$.

${ }^{19}$ This is consistent with the experimentally observed sub-additivity (see Read 2001) in which discounting over a delay is greater when the delay is divided into subintervals than when it is left undivided.

${ }^{20}$ Uncertainty about how/whether subjects will be paid by the experimenter in the future can be mitigated through careful design (for instance, see Andreoni and Sprenger 2012a and Benhabib, Bisin, and Schotter 2010).
} 
Third, in the context of our design, the possibility that some subjects are individually time inconsistent poses an additional challenge for our main experimental goal. Our focus is on how aggregation relates to time inconsistency, and so we wish to begin with time-consistent underlying preferences and measure the inconsistency that emerges from aggregation. Thus, we wish to induce and control the underlying individual preferences to isolate the effects of aggregation.

Finally, subjects' organic time preferences are unpredictable from the experimenter's perspective and could potentially be rather narrow for the time horizons that are feasible in a lab setting (even with delayed payments). ${ }^{21}$ This would limit our ability to test aggregation of an assortment of different time preferences, which is relevant for economic decisions that have long horizons.

Taking all of these points together, directly controlling subjects' individual base preferences becomes essential. This led us to develop a new elicitation technique, aimed at inducing and controlling individual discount factors, while maintaining the nature of the trade-offs featured in aggregation, as we now describe.

\section{B. “As-if” Consumption Streams}

To represent streams of consumption at three different times we used combinations of tokens of three different colors. Instead of a subject receiving some amount of consumption today, some amount $t$ periods from now, and some amount $t^{\prime}$ periods from now, the subject would receive some amount of blue tokens, some amount of red tokens, and some amount of gray tokens. We then induced a discount factor by having different exchange rates for tokens into cash, depending on their color.

To induce a discount factor of $\delta$, we paid a subject one cent for each blue token, $\delta$ cents for each red token, and $\delta^{2}$ cents for each gray token. For instance, a subject with an assigned discount factor of $\delta=0.9$ would get 1 cent for each blue token, 0.9 cents for each red token, and 0.81 cents for each gray token. If, for example, such a subject were faced with a choice between a stream of tokens $C=(105,0,0)$ of blue, red, and gray tokens, respectively (so 105 blue tokens and none of the other colors), and another of $C^{\prime}=(0,160,0)$ (so 160 red tokens), the stream $C$ would be worth 105 cents to the subject and $C^{\prime}$ would be worth $0.9 \times 160=144$ cents. In particular, $C^{\prime}$ would offer a greater overall payoff.

This does not explicitly involve timing. Nonetheless, it involves exactly the same calculations that we assume agents make in standard economic models, and in the experiments this allows us to isolate how subjects aggregate these preferences. In particular, it allows us to gain insights on the impact of a wide range of discount factor combinations, which would be difficult using organic time preferences of subjects as that would require us to either run very large experiments (to get many combinations of discount factors), or introduce many long time horizons to create substantial heterogeneities; and these would still face the difficulties mentioned earlier of eliciting actual time preferences and not other confounds that are very

\footnotetext{
${ }^{21}$ For instance, Andreoni and Sprenger (2012a), who, with monetary rewards, observe insignificant time inconsistency in individual preferences, estimate preferences assuming CRRA utilities. The resulting annual discount rates vary between 0.246 and 0.377 , depending on specification, with standard errors varying between 0.064 and 0.173 . In any reasonable experimental horizon, these estimates would translate into rather narrow regions of discount factors.
} 
likely to be present with long horizons. ${ }^{22}$ Nonetheless, as mentioned, this technique does not involve the explicit time framing and any of its "psychological" cognitive implications. In that sense, our experimental tests are ones inspecting how individuals aggregate sequences of payments that involve discounting, but not a direct test of how people behave when faced with time-based choices. This also allows us to isolate the aggregation issue by imposing actual exponential discounting on the base preferences and then examining biases that result in the aggregation.

Under this construction, the choice between $C$ and $C^{\prime}$ becomes clear as the payoff values to subjects are then simple multiplications of the induced discount factors times the relevant numbers of blue, red, and gray tokens. In order to further focus on aggregation, we also presented the subjects with the net present values rather than the tokens and discount factors, and then checked that choices coincided under the two different presentations. Direct presentation of net present values allows us to avoid behaviors that are driven by simple calculation errors. In particular, to check that these two different presentations were functionally equivalent we ran, as a control, several complementary sessions in which choices were presented as explicit "time" (token) streams with discount factors. Subjects made choices that were statistically indistinguishable across the two types of experimental framings. ${ }^{23}$

\section{Details of the Design}

Subjects made a series of decisions over two alternative consumption "streams," such as the streams $C$ and $C^{\prime}$ described above.

Every subject made a choice in each round between two different consumption streams. These consumption streams were for a group that did not include the chooser. So, the chooser was determining a payoff profile for a group of three subjects, call them members 1,2, and 3. For instance, one of the choices subjects were faced with was between streams $C=(105,0,0)$ and $C^{\prime}=(0,160,0)$. For that choice, if group member 1 was assigned a discount factor of 0.2 and group member 2 a discount factor of 0.9 , then the present discounted values of $C$ and $C^{\prime}$ for these two members would be $U_{1}(C)=U_{2}(C)=105$, and $U_{1}\left(C^{\prime}\right)=32$ and $U_{2}\left(C^{\prime}\right)=144$. Each group also had a third member whose payoff was generally the same across the two choices, in this case set to 80 for both choices. ${ }^{24}$ The chooser

\footnotetext{
${ }^{22}$ Note also that the translation into the exponential discounting setting studied by Koopmans (1960) is straightforward when colored tokens are translated into timed consumption, with the order naturally induced by the tokens' values. For instance, time consistency would imply that $x$ blue tokens are preferred to $y$ red tokens if and only if $x$ red tokens are preferred to $y$ gray tokens.

${ }^{23}$ We ran several auxiliary sessions (with 64 subjects) in which 20 choices were presented as streams of discounted units. These, by and large, correspond to the choices reported upon in the body of the paper in which only subjects for whom the alternatives affected payoffs participated. These auxiliary sessions were run using choices that involved groups of only two individuals (mainly in order to assure the interface was not too loaded with information as each alternative now entailed three numbers, corresponding to the number of tokens of each color, for each subject). In these sessions subjects' choices affected their own payoffs. Subjects could send messages to one another and, upon arriving at a consensus, the corresponding choice was implemented and the resulting total payoffs (the effective net present values) were given. (See instructions in the online materials.) We also ran identical sessions (with 30 subjects), with two subjects arriving at a consensus that was then implemented, in which alternatives were presented as net present values, much as in the paper. Using a test of proportions, none but one of the choices leads to significantly different choices (the only choice leading to a $p$-value lower than 10 percent when the tested hypothesis is that the samples are identical).

${ }^{24}$ The third member was included since we also allowed for some choices inherently related to groups of three in order to check for intransitivities, rather than time consistencies. Those relate to additional theories from Jackson
} 
was faced with a decision between $C$ or $C^{\prime}$, leading to present-discounted payoffs of $(105,105,80)$ from $C$ for the three members of the group, or $(32,144,80)$ for $C^{\prime}$.

In terms of payments, only one member of the group was actually randomly selected to be paid. In particular, we would randomly match two subjects $A$ and $B$. Then $A$ was randomly assigned a role as one of $B$ 's group members (and $B$ was assigned a role as one of some other subject's group). If Subject $A$ was randomly assigned the role of Member $x$, then $A$ was to be paid based on what Member $x$ should receive according to subject $B$ 's allocation choice. Subjects were simply told that their decision would affect a randomly chosen other subject who would be randomly assigned to one of the three roles. Subjects were randomly re-assigned at the end of each round. Subjects were told their cumulative payoffs (and only their own) after each round.

Subjects interacted only through the computerized interface. Two practice rounds were followed by a number of rounds of actual choice (choices are listed in Table 1 below). ${ }^{25}$ Each of the 38 choices subjects faced was comprised of three payoffs, to the three potential members. When comparing two such alternatives, there are three dimensions of the alternatives that may conceivably play an important role in choice: the sum of payoffs, the distribution of payoffs, and the marginal differences between alternatives for each of the subjects. We designed the set of payoffs to vary on these three dimensions.

The experiments were conducted at the California Social Sciences Experimental Laboratory (CASSEL) at UCLA with 60 subjects participating in six separate sessions. Subjects were paid the sum of payoffs throughout the rounds, averaging $\$ 39$, in addition to a show-up fee of $\$ 5.26$

\section{Time Inconsistency}

Several choices subjects faced corresponded to choices between two consumption streams and their delayed version. For instance, suppose group member 1 was assigned a discount factor of 0.2 and group member 2 a discount factor of 0.9 . First, consider a choice between the streams $C=(105,0,0)$ and $C^{\prime}=(0,160,0)$. The present discounted values of $C$ and $C^{\prime}$ for these two members would be $U_{1}(C)=U_{2}(C)=105$, and $U_{1}\left(C^{\prime}\right)=32$ and $U_{2}\left(C^{\prime}\right)=144$. The delayed decision is then between streams $C^{\prime \prime}=(0,105,0)$ versus $C^{\prime \prime \prime}=(0,0,160)$,

and Yariv (2014). The intransitivities appeared quite prevalently and almost exactly in line with the theory (particularly in auxiliary experiments in which agents explicitly voted on which consumption streams would be chosen). For the sake of space we do not report them here; but we kept with three members per group in order to keep the interface similar for the subjects throughout the experiments. For time inconsistencies we only need two different time preferences, and so we simply set the third member's payoffs to be constant across the decision to effectively eliminate that member's payoffs from the decision process.

${ }^{25}$ Our full instructions are available in the online materials.

${ }^{26}$ Choices were shuffled in order to avoid framing effects, and so note that the orders of the decisions in the data in the online materials differ from Table 1. In order to control for the ordering of choices, there were two experimental sessions. In one session, of 36 subjects (that we split into three separate groups for payment purposes, so that behavior is fully independent across them) subjects faced 14 paid choices (in addition to two practice ones). In the second session there were 24 subjects (that we again split into three separate groups for payment purposes). These subjects faced 38 paid choices (in addition to the two practice ones). Furthermore, the 14 paid choices of the first session were the last 14 paid choices in the second session. There were no significant differences between the observed selections made by the two sets of subjects (using proportions tests to compare the choices within the two samples). In what follows we report observations aggregated across these sessions, with most of the analysis based on the 14 choices common across the sessions so that there are 60 subjects. 
Table 1-The List of Choices (in Terms of Present Discounted Utility Profiles) and SubJects' Selections

\begin{tabular}{|c|c|c|c|}
\hline Choice & Alternative 1 & Alternative 2 & Alternative 1 Chosen \\
\hline 1 & $(105,105,80)$ & $(32,144,80)$ & $83 \%$ \\
\hline 2 & $(21,95,80)$ & $(6,130,80)$ & $47 \%$ \\
\hline 3 & $(125,85,80)$ & $(52,124,80)$ & $78 \%$ \\
\hline 4 & $(41,75,80)$ & $(26,110,80)$ & $27 \%$ \\
\hline 5 & $(145,65,80)$ & $(72,104,80)$ & $83 \%$ \\
\hline 6 & $(61,55,80)$ & $(46,90,80)$ & $34 \%$ \\
\hline 7 & $(165,45,80)$ & $(92,84,80)$ & $38 \%$ \\
\hline 8 & $(81,35,80)$ & $(66,70,80)$ & $33 \%$ \\
\hline 9 & $(125,85,80)$ & $(32,144,80)$ & $83 \%$ \\
\hline 10 & $(21,95,80)$ & $(16,120,80)$ & $53 \%$ \\
\hline 11 & $(165,45,80)$ & $(32,144,80)$ & $41 \%$ \\
\hline 12 & $(21,95,80)$ & $(26,100,80)$ & $17 \%$ \\
\hline 13 & $(185,25,80)$ & $(32,144,80)$ & $82 \%$ \\
\hline 14 & $(21,95,80)$ & $(46,90,80)$ & $25 \%$ \\
\hline 15 & $(250,170,80)$ & $(104,248,80)$ & $87 \%$ \\
\hline 16 & $(82,150,80)$ & $(52,220,80)$ & $29 \%$ \\
\hline 17 & $(290,130,80)$ & $(144,208,80)$ & $80 \%$ \\
\hline 18 & $(122,110,80)$ & $(92,180,80)$ & $37 \%$ \\
\hline 19 & $(53,95,80)$ & $(130,40,80)$ & $37 \%$ \\
\hline 20 & $(53,74,80)$ & $(40,78,80)$ & $75 \%$ \\
\hline 21 & $(105,105,105)$ & $(30,210,105)$ & $62 \%$ \\
\hline 22 & $(105,105,105)$ & $(70,170,105)$ & $58 \%$ \\
\hline 23 & $(105,105,105)$ & $(110,130,105)$ & $33 \%$ \\
\hline 24 & $(105,105,105)$ & $(29,245,105)$ & $46 \%$ \\
\hline 25 & $(105,105,105)$ & $(77,197,105)$ & $21 \%$ \\
\hline 26 & $(105,105,105)$ & $(125,149,105)$ & $21 \%$ \\
\hline 27 & $(125,85,125)$ & $(52,124,52)$ & $83 \%$ \\
\hline 28 & $(125,85,85)$ & $(52,124,124)$ & $29 \%$ \\
\hline 29 & $(41,75,41)$ & $(26,110,26)$ & $67 \%$ \\
\hline 30 & $(41,75,75)$ & $(26,110,110)$ & $21 \%$ \\
\hline 31 & $(145,65,65)$ & $(72,104,104)$ & $21 \%$ \\
\hline 32 & $(61,55,61)$ & $(46,90,46)$ & $42 \%$ \\
\hline 33 & $(105,105,150)$ & $(32,144,150)$ & $62 \%$ \\
\hline 34 & $(21,95,150)$ & $(6,130,150)$ & $25 \%$ \\
\hline 35 & $(125,85,150)$ & $(52,124,150)$ & $92 \%$ \\
\hline 36 & $(41,75,150)$ & $(26,110,150)$ & $25 \%$ \\
\hline 37 & $(145,65,150)$ & $(72,104,150)$ & $75 \%$ \\
\hline 38 & $(61,55,150)$ & $(46,90,150)$ & $21 \%$ \\
\hline
\end{tabular}

which induce present discounted utilities of $U_{1}\left(C^{\prime \prime}\right)=21$ and $U_{2}\left(C^{\prime \prime}\right)=95$, and $U_{1}\left(C^{\prime \prime \prime}\right)=6$ and $U_{2}\left(C^{\prime \prime \prime}\right)=130$. In each group we also had a third member whose payoff was often the same across the two choices, in this case 80 for all four choices. ${ }^{27}$ Thus, in terms of profiles of present discounted utilities corresponding to the streams these become $(105,105,80)$ for $C$ and $(32,144,80)$ for $C^{\prime}$; and $(21,95,80)$ for $C^{\prime \prime}$ and $(6,130,80)$ for $C^{\prime \prime \prime}$. In fact, this pair of choices corresponded to choices 1 and 2 in our experiments. A subject would be present biased just with respect to these two choices if he or she picked $C$ over $C^{\prime}$ and then $C^{\prime \prime \prime}$ over $C^{\prime \prime}$ (as more than a third of the subjects did). Similarly, choices 3-6 corresponded to similar pairs in

\footnotetext{
${ }^{27}$ The third member was included since we also allowed for some choices inherently related to groups of three in order to check for intransitivities. The intransitivities appeared quite prevalently and almost exactly in line with the theory (particularly in auxiliary experiments in which agents explicitly voted on which consumption streams would be chosen). For the sake of presentation space we do not report them here; but we kept with three members per group in order to keep the interface similar throughout.
} 


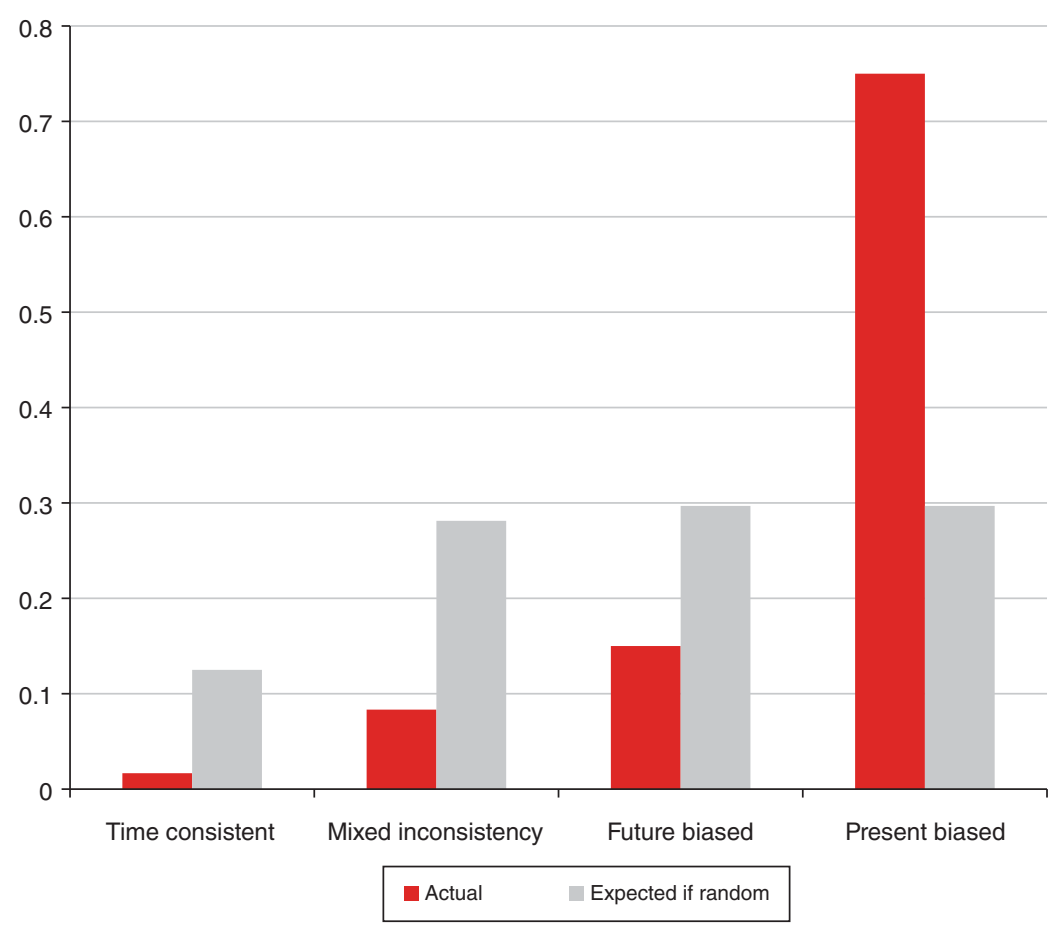

Figure 1. Frequency of Individuals of Different Time Consistency Types

which we considered different initial wealths for members 1 and 2 that were affected by the planner's selection (see Table 1).

These choices allow us to distinguish time-consistent planners (who make corresponding selections in the original and delayed choice problems), present-biased planners (who sometimes choose the immediate smaller consumption, but the larger delayed consumption when all consumption is delayed), future-biased planners (picking delayed consumptions initially, but more immediate rewards when both choices are delayed), and planners who do not fall squarely into any of the above categories.

Figure 1 depicts the distribution of individuals based on this classification. A large majority, 75 percent of individuals, are present biased, while less than 2 percent (only one subject) are time consistent, and the remaining are either future-biased or showing mixed inconsistencies. ${ }^{28}$ These results are very unlikely to arise from subjects randomly making selections. Indeed, Figure 1 also depicts the expected distribution of types were individuals selecting each choice in a pair with a probability of 0.5 , which is significantly different from the observed distribution with any reasonable levels of statistical confidence. 29

\footnotetext{
${ }^{28}$ These categories were based on comparing decisions where the same decisions were made with different time perspectives. Present bias was assigned if a subject sometimes made decisions which exhibited present bias (for example, picking $C$ over $C^{\prime}$ and then $C^{\prime \prime \prime}$ over $C^{\prime \prime}$ in the above described choices which were in the decision set) and never the reverse. Future bias indicated that the reverse was true, so that subjects sometimes chose the reverse of the present biased decisions and never chose in a present biased manner. Time consistency indicates that the same decisions were always made regardless of whether the alternatives in a choice were delayed; and mixed inconsistencies captures the remaining subjects.

${ }^{29}$ Furthermore, the probability of achieving at least 75 percent present-biased individuals under random choice is of the magnitude of $10^{-28}$.
} 
TABle 2-Choices Distinguishing Between

Three Prominent Collective Utility Functions

\begin{tabular}{llccc}
\hline \hline & & & & \\
& & Profile of payoffs & $\begin{array}{c}\text { Types of planner } \\
\text { preferences preferring }\end{array}$ & Percent choosing \\
\hline Choice 1 & C & $(105,105,80)$ & M, I, U & 83 \\
& $\mathrm{C}^{\prime}$ & $(32,144,80)$ & & 17 \\
Choice 2 & $\mathrm{C}$ & $(21,95,80)$ & M, I & 47 \\
& $\mathrm{C}^{\prime}$ & $(6,130,80)$ & $\mathrm{U}$ & 53 \\
Choice 3 & $\mathrm{C}$ & $(61,55,150)$ & $\mathrm{M}$ & 21 \\
& $\mathrm{C}^{\prime}$ & $(46,90,150)$ & I, U & 79 \\
Choice 4 & $\mathrm{C}$ & $(21,95,80)$ & & 17 \\
& $\mathrm{C}^{\prime}$ & $(26,100,80)$ & M, I, U & 83 \\
\hline
\end{tabular}

Note: (U1, U2, U3); M = Maximin; I = Inequality; $\mathrm{U}=$ Utilitarian

\section{E. Social Planners' Collective Utility Functions}

As mentioned, the choices we offered subjects were designed to distinguish the ways in which planners made choices, including pairs of consumption streams that varied in the sum of payoffs, the distribution of payoffs, and the marginal differences between alternatives.

Specifically, we designed the choice problems to distinguish between the three most prominent ways from the welfare literature in which planners might evaluate alternatives: based on the sum of utilities across agents (utilitarianism), based on maximizing the minimum utility (maximin or a "Rawlsian" approach), or based on some weighting of the distribution of payoffs (some form of egalitarianism), say the minimization of the standard deviation of payoffs. ${ }^{30}$

Table 2 illustrates how the choices faced by subjects distinguish these motives. Table 2 contains four choices that differed in terms of which selection a subject would make if they were using a different sort of collective utility function, the type of objective that would lead to a selection of either alternative, and the corresponding experimental frequencies with which the first alternative was selected. ${ }^{31}$

The table illustrates that when all three objectives lead to the same alternatives (the first and fourth choice pairs), most of the subjects followed those prescriptions (83 percent in both cases). In the case where the prescriptions under utilitarianism and egalitarianism diverge (Choice 2), there is a substantial split in the decisions of the subjects. However, in the choice that differed only in the prescription of maximin (Choice 3) compared to utilitarianism and inequality aversion, the pattern looks (statistically) indistinguishable from the case where all three lead to the same alternative (Choice 4). Indeed, as the bottom two choices of Table 2 illustrate, the choices in which both utilitarian and egalitarian motives would push agents toward selecting the second alternative, but maximin motives would generate diverse decisions, differed in only 4 percent of subjects. ${ }^{32}$ Thus, in what follows, we consider collective utility functions that depend on the sum and distribution of payoffs.

\footnotetext{
${ }^{30}$ The analysis that follows does not change qualitatively if we consider egalitarian motives that depend on the variance or absolute differences of payoffs.

${ }^{31}$ These choices correspond to choices 1,2,38, and 12 in our design, see Table 1.

${ }^{32}$ Aggregate discrete-choice regression analysis in which selections were explained by the sum of payoffs, the variance of payoffs, and the maximin of payoffs, led to similar observations regarding the relative importance of the sum and variance of payoffs.
} 
A family of collective utility functions that incorporates utilitarianism and inequality aversion is one that evaluates an alternative according to

$$
a \times \text { Mean of Payoffs }-(1-a) \times \text { Standard Deviation of Payoffs, }
$$

where $a \in[0,1]$. Thus, $a$ serves as a weight of how much a planner cares about utilitarian concerns relative to distributional ones. ${ }^{33}$

For each subject and for each specification of the parameter $a$, we can calculate the fraction of decisions that would be implied by the maximization of (2) and coincide with that subject's observed selections. We call that fraction the individual score. For each $a$, the overall score is the average over all individual subject scores.

We allow for heterogeneity in the $a$ parameters that best describe subjects. In particular, we considered different numbers of "types" that describe the subjects. A type is characterized by a range of parameters $a$ that all lead to the same predictions (given the finite number of alternatives, not all $a$ parameters are distinguished). So, if we just allow for one type, we find the single $a$ that best fits all of the decisions by all of the subjects. If we allow for two types, we look for two different $a$ parameters that best describe the decisions of all of the subjects, when each subject must be assigned to one of the two different $a$ parameters and so forth. So, for each number of types $k$, we look for a partition of the subject population into $k$ groups and a value of $a$ for each group in the partition that maximizes overall scores. Table 3 presents the values of the best fitting (ranges of) $a$ parameters for each number of types $k$, and the corresponding average score for individuals of each type.

In terms of preference parameters, assuming all subjects have the same collective utility function leads to an estimated parameter $a$ of 0.75 .34 However, allowing for more than one type of collective utility function among the subjects leads to a substantial fraction of individuals (between 15 percent-25 percent) who put very little weight on utilitarian concerns and care mostly about the variation in payoffs. Regardless of the number of types fitted, more than three quarters of the population has a taste parameter $a$ that is 0.7 or higher (using the midpoint if an interval of parameters fits equally well for a subject). Thus, although most subjects are best fit by having some weight on inequality, many weight it in a minor way. Nonetheless, a minority of subjects appear to weight inequality much more substantially. We note, however, that the types with lower utilitarian incentives were characterized by a lower overall score, suggesting that our model of planners' objectives better fits subjects who place greater weight on utilitarian motives. ${ }^{35}$

In Figure 2, we present the best fitting score for each type, as dependent on the number of types. When the possible number of types is 60 (the number of

\footnotetext{
${ }^{33}$ As mentioned before, the results are quantitatively similar when using the sum of absolute differences between payoffs instead of the standard deviation of payoffs, and both offer slightly better fits than those derived by using variance instead of standard deviation, which is more nonlinear in the level of inequality.

${ }^{34}$ We note that if subjects only cared about efficiency, then $a=1$, corresponding to a score of about 71 percent, or approximately 29 percent probability of errors in prediction. If subjects only cared about the standard deviation of payoffs, then $a=0$, and the score would fall to around 49 percent (approximately the score one would expect from random choice), corresponding to 51 percent prediction errors.

${ }^{35}$ The score is still substantially higher than the 50 percent expected score generated by random selections. We note that it is possible that some subjects had objectives that accounted for multiple choices made, rather than viewing each choice on the margin. Although much of the variation in behavior is captured by objective functions that consider each choice in isolation, broader objectives are an important consideration for further research.
} 
Table 3-Best Fitting Utilitarian-Egalitarian Preferences by Number of Types

\begin{tabular}{|c|c|c|c|c|c|c|c|}
\hline Number of types & & Type 1 & Type 2 & Type 3 & Type 4 & Type 5 & Type 6 \\
\hline 1 & $\begin{array}{l}\text { Best fit " } a \text { " range } \\
\text { Percent of this type } \\
\text { Score }\end{array}$ & $\begin{array}{c}0.75 \\
(100) \\
0.72\end{array}$ & & & & & \\
\hline 2 & $\begin{array}{l}\text { Best fit " } a \text { " range } \\
\text { Percent of this type } \\
\text { Score }\end{array}$ & $\begin{array}{c}0-0.25 \\
(25) \\
0.67\end{array}$ & $\begin{array}{c}0.95-1 \\
(75) \\
0.80\end{array}$ & & & & \\
\hline 3 & $\begin{array}{l}\text { Best fit " } a \text { " range } \\
\text { Percent of this type } \\
\text { Score }\end{array}$ & $\begin{array}{c}0-0.25 \\
(21) \\
0.66\end{array}$ & $\begin{array}{l}0.75 \\
(40) \\
0.79\end{array}$ & $\begin{array}{c}0.95-1 \\
(39) \\
0.86\end{array}$ & & & \\
\hline 4 & $\begin{array}{l}\text { Best fit " } a \text { " range } \\
\text { Percent of this type } \\
\text { Score }\end{array}$ & $\begin{array}{c}0-0.25 \\
(17) \\
0.66\end{array}$ & $\begin{array}{l}0.7 \\
(18) \\
0.82\end{array}$ & $\begin{array}{l}0.75 \\
(27) \\
0.81\end{array}$ & $\begin{array}{c}0.95-1 \\
(38) \\
0.86\end{array}$ & & \\
\hline 5 & $\begin{array}{l}\text { Best fit " } a \text { " range } \\
\text { Percent of this type } \\
\text { Score }\end{array}$ & $\begin{array}{l}0-0.25 \\
(15) \\
0.6449\end{array}$ & $\begin{array}{l}0.55 \\
(7) \\
0.7968\end{array}$ & $\begin{array}{l}0.7 \\
(14) \\
0.838\end{array}$ & $\begin{array}{l}0.75 \\
(26) \\
0.814\end{array}$ & $\begin{array}{l}0.95-1 \\
(38) \\
0.8653\end{array}$ & \\
\hline 6 & $\begin{array}{l}\text { Best fit " } a \text { " range } \\
\text { Percent of this type } \\
\text { Score }\end{array}$ & $\begin{array}{c}0-0.25 \\
(15) \\
0.64\end{array}$ & $\begin{array}{l}0.55 \\
(7) \\
0.80\end{array}$ & $\begin{array}{l}0.7 \\
(13) \\
0.84\end{array}$ & $\begin{array}{l}0.75 \\
(25) \\
0.82\end{array}$ & $\begin{array}{l}0.85 \\
(17) \\
0.88\end{array}$ & $\begin{array}{c}0.95-1 \\
(23) \\
0.85\end{array}$ \\
\hline
\end{tabular}

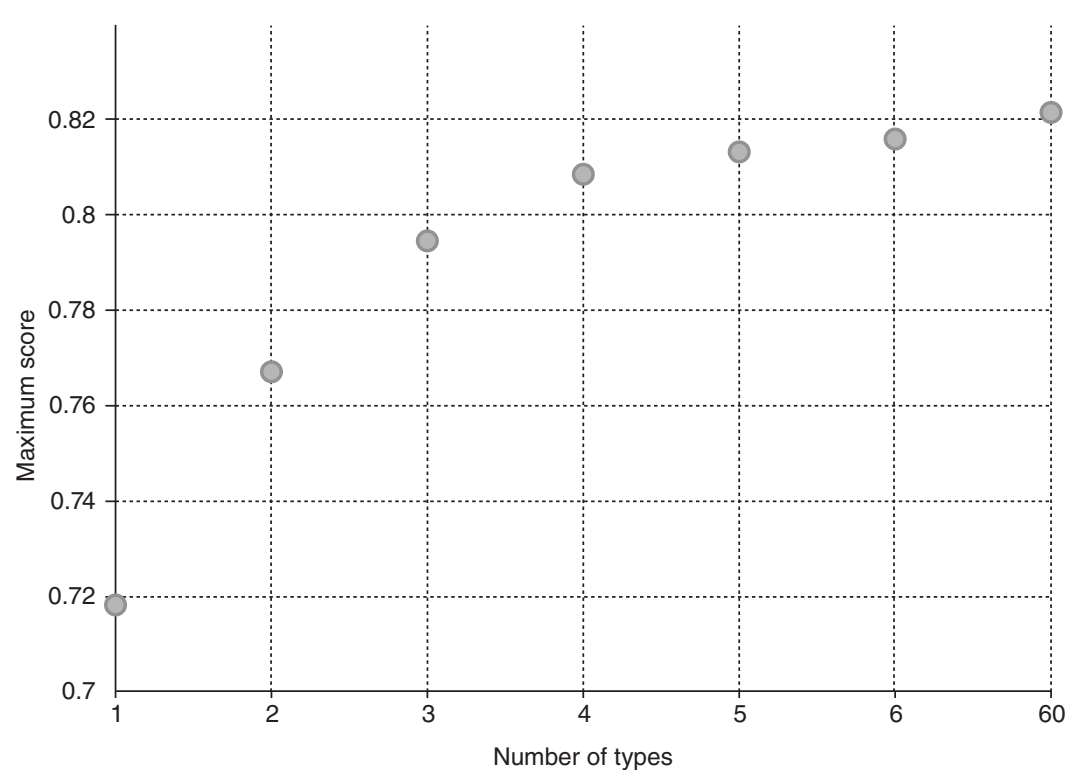

Figure 2. Score as a Function of the Number of Types

subjects), each subject could have an individual $a$, and so the derived score is the maximal feasible one under this model and is 82 percent. As can be seen in the figure, allowing for 5 or 6 types yields scores that are not substantially lower. On the other extreme, assuming the population is completely homogeneous, leads to a lower score of approximately 72 percent .

To conclude, our experimental results are in line with the theory in that, with almost no exceptions (59 out of 60) experimental planners are time inconsistent, with three quarters of them exhibiting choices that are present biased. Furthermore, planners in these experiments generally act in ways that are consistent with a 
combination of utilitarian and equality-sensitive motives, with a large fraction putting a substantial weight on the utilitarian attributes of alternatives. Recall that Proposition 1 implied that whenever utilitarian motives guide decisions, behavior will be present biased. In that sense, the preference characterization of our experimental social planners is in line with the prevalent present bias observed in the lab.

\section{Concluding Remarks}

Our starting point is the theoretical observation that whenever there is some heterogeneity in the population, utilitarian aggregation exhibits present bias, where choices appear to correspond to increasing patience over time. These results are confirmed by an array of experimental results. Social planners in the lab put high weights on utilitarian motives and, in accordance, three quarters of subjects exhibit time inconsistencies that take the form of present bias. Thus, utilitarian policy makers who care about some proxy of (utilitarian) efficiency will be present biased. In addition, the results suggest that even when estimated preferences pertaining to groups (say, households) exhibit time inconsistencies, they may arise from heterogeneous but time-consistent individual preferences. Therefore, welfare maximization requires a careful analysis with the primitive preferences taken into account, rather than a simple substitution with a non-existent representative agent.

APPENDIX

\section{A. Proof of Proposition 1}

Without loss of generality, we normalize all utility functions so that $u_{i}(0)=0$ for all $i$.

We prove a stronger version of Proposition 1.

A collective utility function is a generalized weighted utilitarian function if there exist weights $w_{i} \in[0,1]$ such that $\sum_{i} w_{i}=1$ and

$$
V(C)=F\left(\sum_{i} w_{i} U_{i}(C)\right)
$$

where $F$ is an increasing function.

PROPOSITION $1 *$ : If $u_{i}=u$ for all $i$ where $u$ is continuous and strictly increasing, and $V$ is a generalized weighted utilitarian function with weights $w_{i}>0$ and $w_{j}>0$ for at least two agents $i$ and $j$ such that $\delta_{i} \neq \delta_{j}$, then $V$ is present-biased.

\section{PROOF OF PROPOSITION 1*:}

By the definition of a generalized weighted utilitarian function and the conditions of the proposition, we can write

$$
V(C[x, t])=F\left(\sum_{i} w_{i} \delta_{i}^{t-1} u(x)\right)
$$


To see the first part of the definition of present-bias, note that $V\left(C_{x}^{t}\right) \geq V\left(C_{y}^{t+k}\right)$ implies that

$$
\sum_{i} w_{i} \delta_{i}^{t-1} u(x) \geq \sum_{i} w_{i} \delta_{i}^{t+k-1} u(y)
$$

If $u(x)=0$ then $u(y)=0$ and from monotonicity, $x=y=0$ and all time evaluations are identical. Suppose then that $u(x)>0$. The above inequality can be written as

$$
\frac{\sum_{i} w_{i} \delta_{i}^{t-1}}{\sum_{i} w_{i} \delta_{i}^{t+k-1}} \geq \frac{u(y)}{u(x)}
$$

We show that

$$
\frac{\sum_{i} w_{i} \delta_{i}^{t-1}}{\sum_{i} w_{i} \delta_{i}^{t+k-1}}
$$

is decreasing in $t$.

Note that the derivative of this expression is

$$
\frac{\left(\sum_{i} w_{i} \ln \left(\delta_{i}\right) \delta_{i}^{t-1}\right)\left(\sum_{i} w_{i} \delta_{i}^{t+k-1}\right)-\left(\sum_{i} w_{i} \ln \left(\delta_{i}\right) \delta_{i}^{t+k-1}\right)\left(\sum_{i} w_{i} \delta_{i}^{t-1}\right)}{\left(\sum_{i} w_{i} \delta_{i}^{t}\right)^{2}} .
$$

The numerator can be written as

$$
\sum_{i} \sum_{j} \ln \left(\delta_{i}\right) w_{i} w_{j} \delta_{i}^{t-1} \delta_{j}^{t-1}\left(\delta_{j}^{k}-\delta_{i}^{k}\right)
$$

which we can rewrite as

$$
\sum_{i, j: i<j} w_{i} w_{j} \delta_{i}^{t-1} \delta_{j}^{t-1}\left(\ln \left(\delta_{i}\right)-\ln \left(\delta_{j}\right)\right)\left(\delta_{j}^{k}-\delta_{i}^{k}\right)
$$

and each of the expressions in this summand is positive whenever $w_{i} w_{j} \delta_{i}^{t-1} \delta_{j}^{t-1} \neq 0$ and $\delta_{j} \neq \delta_{i}$, and is zero otherwise.

This implies that

$$
\frac{\sum_{i} w_{i} \delta_{i}^{t-2}}{\sum_{i} w_{i} \delta_{i}^{t+k-2}} \geq \frac{u(y)}{u(x)}
$$

or that $V\left(C_{x}^{t-1}\right) \geq V\left(C_{y}^{t+k-1}\right)$ and so the first part of the definition of present-bias is satisfied.

Next, given that $u$ is continuous and increasing, and $u(0)=0$ (recall that all utility functions are so normalized), we can find $x$ and $y$ such that

$$
u(x)=\sum_{i} w_{i} \delta_{i}^{k} u(y)
$$


Given that $w_{i}>0$ and $w_{j}>0$ for some $\delta_{i} \neq \delta_{j}$, it follows, as shown already, that

$$
\frac{\sum_{i} w_{i} \delta_{i}^{t}}{\sum_{i} w_{i} \delta_{i}^{t+1}}
$$

is strictly decreasing in $t$. Therefore, iterative application of the above implies that

$$
\sum_{i} w_{i} \delta_{i}^{t} u(x)<\sum_{i} w_{i} \delta_{i}^{t+k} u(y)
$$

for all $t \geq 1$. By continuity of $u$, we can then find some $\varepsilon$ such that

$$
u(x+\varepsilon)>\sum_{i} w_{i} \delta_{i}^{k} u(y) \quad \text { and } \quad \sum_{i} w_{i} \delta_{i}^{t} u(x+\varepsilon)<\sum_{i} w_{i} \delta_{i}^{t+k} u(y)
$$

for all $t \geq 1$. This establishes the second part of the definition of present-bias.

\section{REFERENCES}

Abdellaoui, Mohammed, Oliver l'Haridon, and Corina Paraschiv. 2010. "Individual vs. Collective Behavior: An Experimental Investigation of Risk and Time Preferences in Couples." HEC Paris Research Paper No. 944/2011.

Ainslie, George. 1975. "Specious Reward: A Behavioral Theory of Impulsiveness and Impulse Control." Psychological Bulletin 82 (4): 463-96

-Amador, Manuel, Iván Werning, and George-Marios Angeletos. 2006. "Commitment vs. Flexibility." Econometrica 74 (2): 365-96.

Ambrus, Attila, and Kareen Rozen. 2014. "Rationalising Choice with Multi-self Models." Economic Journal. doi: 10.1111/ecoj.12103.

Andreoni, James, and John Miller. 2002. "Giving According to GARP: An Experimental Test of the Consistency of Preferences for Altruism.” Econometrica 70 (2): 737-53.

Andreoni, James, and Charles Sprenger. 2012a. "Estimating Time Preferences from Convex Budgets." American Economic Review 102 (7): 3333-56.

Andreoni, James, and Charles Sprenger. 2012b. "Risk Preferences Are Not Time Preferences." American Economic Review 102 (7): 3357-76.

Bénabou, Roland, and Jean Tirole. 2004. "Willpower and Personal Rules.” Journal of Political Economy 112 (4): 848-87.

Benhabib, Jess, Alberto Bisin, and Andrew Schotter. 2010. "Present-Bias, Quasi-hyperbolic Discounting, and Fixed Costs." Games and Economic Behavior 69 (2): 205-23.

Bernheim, B. Douglas. 1999. "Comment on 'Family Bargaining and Retirement Behavior." In Behavioral Dimensions of Retirement Economics, edited by Henry J. Aaron, 273-81. Washington, DC: Brookings Institution Press.

-Bernheim, B. Douglas, and Antonio Rangel. 2004. "Addiction and Cue-Triggered Decision Processes." American Economic Review 94 (5): 1558-90.

Beshears, John, James J. Choi, David Laibson, and Brigitte C. Madrian. 2008. "The Importance of Default Options for Retirement Saving Outcomes: Evidence from the USA." In Lessons from Pension Reform in the Americas, edited by Stephen J. Kay and Tapen Sinha, 59-87. Oxford: Oxford University Press.

-Blackorby, Charles, Walter Bossert, and David Donaldson. 2005. "Temporal Consistency.” In Population Issues in Social Choice Theory, Welfare Economics, and Ethics, 272-85. Cambridge: Cambridge University Press.

Brocas, Isabelle, and Juan D. Carrillo. 2008. "The Brain as a Hierarchical Organization.” American Economic Review 98 (4): 1312-46.

Browning, Martin. 2000. “The Saving Behaviour of a Two-Person Household." Scandinavian Journal of Economics 102 (2): 235-51.

Caplin, Andrew, and John Leahy. 2004. "The Social Discount Rate." Journal of Political Economy 112 (6): 1257-68. 
Charness, Gary, and Matthew Rabin. 2002. "Understanding Social Preferences with Simple Tests." Quarterly Journal of Economics 117 (3): 817-69.

Cherepanov, Vadim, Timothy Feddersen, and Alvaro Sandroni. 2013. "Rationalization." Theoretical Economics 8 (3): 775-800.

Dasgupta, Partha, and Eric Maskin. 2005. "Uncertainty and Hyperbolic Discounting." American Economic Review 95(4): 1290-99.

DellaVigna, Stefano, and Ulrike Malmendier. 2006. "Paying Not to Go to the Gym." American Economic Review 96 (3): 694-719.

Drefahl, Sven. 2010. "How Does the Age Gap between Partners Affect Their Survival?" Demography 47 (2): 313-26.

-Engelmann, Dirk, and Martin Strobel. 2004. "Inequality Aversion, Efficiency, and Maximin Preferences in Simple Distribution Experiments." American Economic Review 94 (4): 857-69.

Farmer, J. Doyne, and John Geanakoplos. 2009. "Hyperbolic Discounting is Rational: Valuing the Far Future with Uncertain Discount Rates.” Cowles Foundation for Research in Economics Discussion Paper No. 1719.

-Feldstein, M. S. 1964. "The Social Time Preference Discount Rate in Cost Benefit Analysis.” Economic Journal 74 (294): 360-79.

Fisman, Raymond, Shachar Kariv, and Daniel Markovits. 2007. "Individual Preferences for Giving." American Economic Review 97 (5): 1858-76.

Frederick, Shane, George Loewenstein, and Ted O’Donoghue. 2002. "Time Discounting and Time Preference: A Critical Review.” Journal of Economic Literature 40 (2): 351-401.

Fudenberg, Drew, and David K. Levine. 2006. "A Dual-Self Model of Impulse Control." American Economic Review 96 (5): 1449-76.

Glimcher, Paul W., and Aldo Rustichini. 2004. "Neuroeconomics: The Consilience of Brain and Decision." Science 306 (5695): 447-52.

Gollier, Christian, and Martin L. Weitzman. 2010. "How Should the Distant Future Be Discounted When Discount Rates Are Uncertain?" Economics Letters 107 (3): 350-53.

Gollier, Christian, and Richard Zeckhauser. 2005. "Aggregation of Heterogeneous Time Preferences." Journal of Political Economy 113 (4): 878-96.

Green, Jerry R., and Daniel A, Hojman. 2009. "Choice, Rationality and Welfare Measurement." http:// www.hks.harvard.edu/fs/dhojman/papers/CRW.pdf.

Hare, Todd A., Colin F. Camerer, and Antonio Rangel. 2009. "Self-Control in Decision-Making Involves Modulation of the vmPFC Valuation System.” Science 324 (5927): 646-8.

Herrnstein, Richard J. 1961. "Relative and Absolute Strength of Response as a Function of Frequency of Reinforcement." Journal of Experimental Analysis of Behavior 4 (3): 267-72.

Hertzberg, Andrew. 2012. "Exponential Individuals, Hyperbolic Households." https://www0.gsb. columbia.edu/faculty/ahertzberg/HH_Paper_July_23_2012.pdf.

Jackson, Matthew O., and Leeat Yariv. 2014. "Collective Dynamic Choice: The Necessity of Time Inconsistency.” http://dx.doi.org/10.2139/ssrn.1699444.

Jackson, Matthew O., and Leeat Yariv. 2014. "Present Bias and Collective Dynamic Choice in the Lab: Dataset.” American Economic Review. http://dx.doi.org/10.1257/aer.104.12.4184.

- Jamison, Dean T., and Julian Jamison. 2011. "Characterizing the Amount and Speed of Discounting Procedures" Journal of Benefit-Cost Analysis 2 (2): 1-56.

Koopmans, T. C. 1960. "Stationary Ordinal Utility and Impatience." Econometrica 28 (2): 287-309.

Kuhn, Michael A. 2013. "Curing the Calorie Crunch: The Effect of EBT on Household Present-Bias." http://econweb.ucsd.edu/ mkuhn/pdfs/k_11-9-13.pdf.

-Laibson, David. 1997. "Golden Eggs and Hyperbolic Discounting." Quarterly Journal of Economics $112(2): 443-77$.

Marglin, S. A. 1963. "The Social Rate of Discount and the Optimal Rate of Investment." Quarterly Journal of Economics 77 (1): 95-111.

Mazzocco, Maurizio. 2007. "Household Inter Temporal Behaviour: A Collective Characterization and a Test of Commitment.” Review of Economic Studies 74 (3): 857-95.

McClure, Samuel M., Keith M. Ericson, David I. Laibson, George Loewenstein, and Jonathan D. Cohen. 2007. "Time Discounting for Primary Rewards." Journal of Neuroscience 27 (21): 5796-804.

McClure, Samuel M., David I. Laibson, George Loewenstein, and Jonathan D. Cohen. 2004. "Separate Neural Systems Value Immediate and Delayed Monetary Rewards.” Science 306 (5695): 503-7.

O'Donoghue, Ted, and Matthew Rabin. 1999. "Doing It Now or Later." American Economic Review 89 (1): 103-24.

Rachlin, Howard. 2000. The Science of Self-Control. Cambridge, MA: Harvard University Press.

Read, Daniel. 2001. "Is Time-Discounting Hyperbolic or Subadditive?" Journal of Risk and Uncertainty 23 (1): 5-32. 
Rode, Ashwin. 2011. "Literature Review: Non-Unitary Models of the Household (Theory and Evidence)."http://www.econ.ucsb.edu/ pjkuhn/Ec250A/StudentsPapers/RodeNonUnitaryModels.pdf.

Schaner, Simone. 2013. "Do Opposites Detract? Intrahousehold Preference Heterogeneity and Inefficient Strategic Savings.” http://www.russellsage.org/sites/all/files/Schaner_Opposites.pdf.

-Souzou, P. D. 1998. “On Hyperbolic Discounting and Uncertain Haard Rates.” Proceedings of the Royall Society of London B 265 (1409): 2015-20.

-Strotz, R. H. 1955. "Myopia and Inconsistency in Dynamic Utility Maximization." Review of Economic Studies 23 (3): 165-80.

Thaler, Richard. 1981. "Some Empirical Evidence on Dynamic Inconsistency." Economics Letters 8 (3): 201-7.

Thaler, Richard H., and H. M. Shefrin. 1981. "An Economic Theory of Self-Control.” Journal of Political Economy 89 (2): 392-406.

United Nations. 2008. Demographic Year Book 2008. New York: Department of Economic and Social Affairs, United Nations.

-Weitzman, Martin L. 2001. “Gamma Discounting.” American Economic Review 91 (1): 260-71.

Xue, Licun. 2008. "The Bargaining Within.” Economics Letters 101 (2): 145-47.

Zuber, Stephane. 2011. “The Aggregation of Preferences: Can We Ignore the Past?" Theory and Decision 70 (3): 367-84. 